\title{
The study of rapid prototyping process with embedded functional inserts
}

\author{
Y.S. Liao, H.C. Li*, M.T. Chen \\ Department of Mechanical Engineering, National Taiwan University, No. 1, Sec. 4, Roosevelt Road, Taipei 106, Taiwan, ROC
}

\begin{abstract}
The purpose of this paper is to study the process of making functional inserts embedded rapid prototyping (RP) parts. A generalized procedure and its associated insert classification method for easy inserting functional parts into a RP product on-line are proposed. The inner product of two vectors, the mock-up construction direction and the surface normal vector of the insert, are taken as the criterion of implementation. Based on this method, most of the axis-symmetric functional inserts are classified into three groups according to their shapes and orientations in the RP part, namely if they can be inserted directly, a simple shape converter is needed, or a multiple shape converter is needed. The shape converter is manufactured in advance if it is required. The shape converter is assembled with the insert, and the whole unit is then placed into the frame. Thereafter, laminating process is proceeded until a complete RP part is finished without any interference. The proposed procedure and insert classification method are verified via the new on-line de-cubing laminated object manufacturing (LOM) process. Three cases studies were considered and experiments were conducted. Results show that the RP parts with insert or functional insert are made successfully.
\end{abstract}

(C) 2007 Elsevier B.V. All rights reserved.

Keywords: Rapid prototyping; Insert; On-line de-cubing

\section{Introduction}

The application of rapid prototyping (RP) technology can reduce the time spent in product development and the errors in mould manufacturing. As a result, the cost is reduced and the product is made more competitive in the market. It is particularly suitable for the many varieties but low-volume manufacturing requirement, short life cycle products as well as customized complicated products commonly encountered in nowadays industry. Considering the products used in the daily life, it is reasonable to expand this technology toward making functional inserts embedded RP parts.

Kataria and Rosen [1] proposed an approach to apply stereolithography apparatus (SLA) with photopolymer to build the complex mechanism, which can solve the problems encountered in the manufacturing procedures, such as structure support, the interference between recoating and insert, laser shadowing, etc. Kathryn, Kong and Mavroidis [2,3] used SLA with material of liquid photopolymer for embedding structure parts. This approach can complete a prototype with the features of com-

\footnotetext{
* Corresponding author. Tel.: +886 2 23660443; fax: +886233664513.

E-mail addresses: liaoys@ntu.edu.tw (Y.S. Liao),d89522019@ntu.edu.tw (H.C. Li), r91522707@ntu.edu.tw (M.T. Chen).
}

plex functional inserts and linkages in one step. However, there are inherent disadvantages of the SLA method to produce the insert part. The complicated and tedious process of sealing and leakage-preventing should be taken in advance for the sensitive parts such as motors, conducting wires, etc., to prevent them from functional failure caused by permeation of liquid photopolymer.

Weiss and Prinz [4] utilized shape deposition manufacturing (SDM) together with milling process to make multi-materials or insert embedded part. This approach was applied by Hatanaka and Cutkosky [5] to embed the elastic insert into the prototype. However, the induced thermal effect on the insert from the deposited material or the bubble vacancy problem still remains unsolved. Besides, the facility for milling process brings about extra production cost. The fused deposition manufacturing (FDM) has been applied recently as well for the same purpose. There are similar difficulties as encountered by the use of SDM for making insert embedded RP part.

Conventional laminated object manufacturing (LOM) cannot perform de-cubing process on-line. Liao and Chiu [6] proposed a new LOM process which can de-cube $30-80 \%$ of waste material on-line, and can fabricate the hollow prototype that leaves the room for insert part. This feature is very appropriate for the fabrication of insert embedded RP part, and the approach will be described in the following sections. 


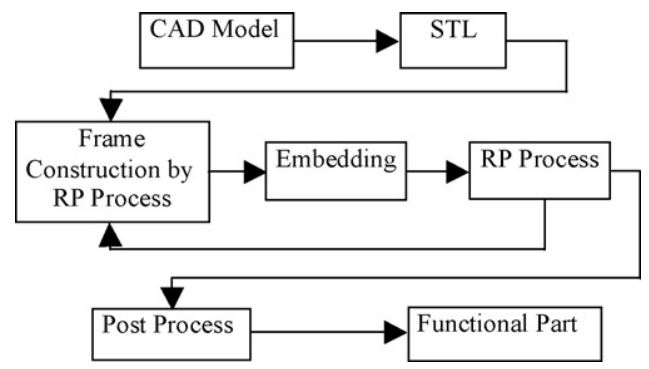

Fig. 1. Basic process for insert part.

\section{The fabrication procedure of inserts}

The RP models with embedded inserts are more than just shape consideration but are given with some functions such as assembly, rotation, translation or transmission by including a solid functional part like the bearing, gear, battery or conducting wire, etc. In order to complete a RP model with insert part, the appropriate process to make insert part to coordinate with the various current RP technologies is highly demanded. Most importantly, the appropriate decomposition criterion to deduce the mock-up frame and the shape converter enables the smooth embedding process of the insert part.

\subsection{The concept of shape converter}

In general, the traditional insert embedded process includes a pre-designed mock-up frame for insert part to avoid problems of laser shadowing and rolling wheel inference due to the complex geometry. The conceptual process is shown in Fig. 1. But the insert parts often possess some complex shapes that prevent the above-mentioned process from being applied directly. For example, the mismatch of the embedding space and the insert shape induces the vacancy problem which blocks the succeeding RP process. Fig. 2(a) shows the vacancy exits between the insert part and the mock-up frame at the upper part, and Fig. 2(b) demonstrates the mismatch of the concave insert part and the succeeding RP process.

The fabrication difficulties will occur according to the specific features of the shape of insert. This problem can be solved by introducing a set of suitable shape converters [1]. Such shape converters can effectively contain the insert and closely embedded with the frame of prototype. Different complicated shapes

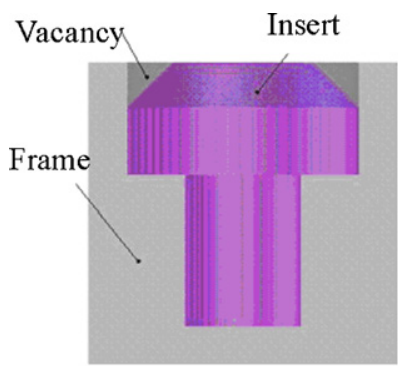

(a)

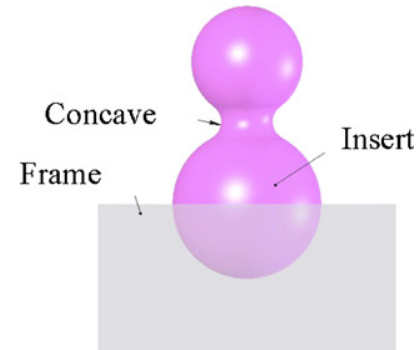

(b)
Fig. 2. (a) The vacancy between the insert part and the mock-up frame, (b) the mismatch from the concave insert part.

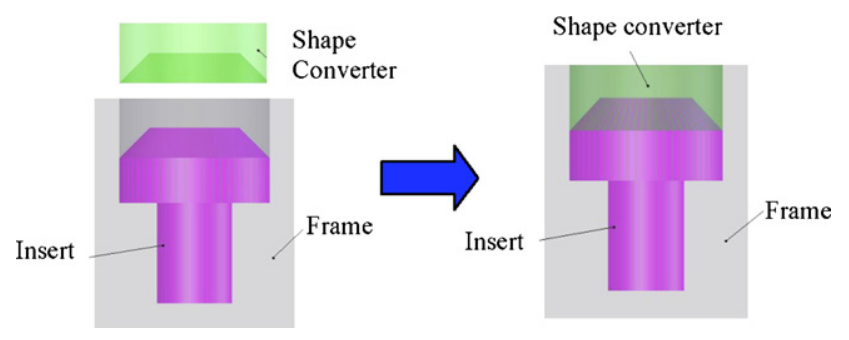

Fig. 3. Elimination of clearance with simple shape converter.

of inserts will require different shape converters. In the paper the shape converter is classified into simple type and split type based on the insert geometry and its insertion direction. A simple type converter uses the feature of insert shape as the basis to fabricate the single shape mold of the insert which can be tightly embedded with the frame. Meanwhile, the split shape converter incorporates more than two pieces of shape converters to engage with insert and the frame to complete the insertion process. The use of simple shape converter can alleviate the problem of vacancy as shown in Fig. 3, and the problem of calabash indention can be solved by a split shape converter as depicted in Fig. 4.

\subsection{Disassembly rules and classification methods}

This paper uses insertion direction and the shape features of insert to establish a set of disassembly and classification rules to design suitable shape converters. The criterion and disassembly line are used to judge if a shape converter is required. The shape converter and frame can be obtained from the geometry of insert by these rules.

\subsubsection{Criterion and disassembly line}

First of all, a criterion to distinguish the shape features as given in Eq. (1) is defined, where in the equation $\vec{B}$ is the unit vector of RP fabrication direction, and $\vec{n}$ is the unit vector normal to the STL rectangular mesh transforming from 3D solid model. The problems of vacancy and the calabash indention as mentioned previously can be detected by means of the inner product between $\vec{B}$ and $\vec{n}$. As shown in Fig. 5 the insert can be successfully inserted when $S=0$, a vacancy will occur when $S<0$, and there is an indention after insertion when $S>0$. From these pictures, it is readily understood that a shape converter and disassembly line are needed when the vacancy and indention occur,

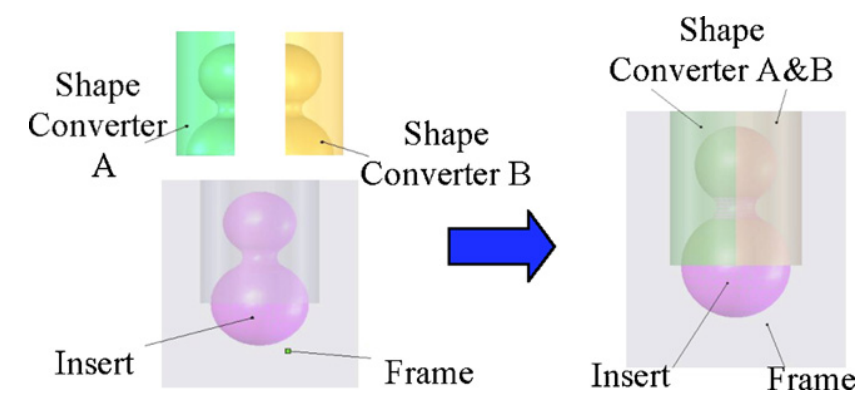

Fig. 4. The shape converter for the concave structure. 


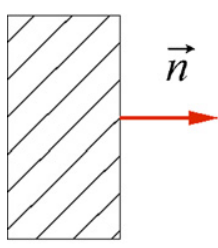

(a)

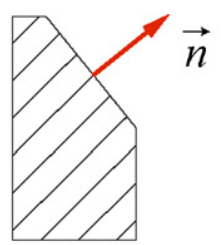

(b)

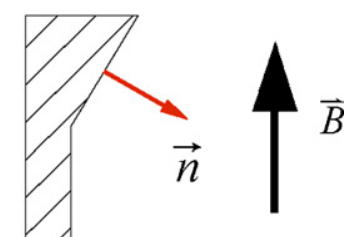

(c)
Fig. 5. The criterion for (a) $S=0$; successful inserting, (b) $S>0$; generating clearance, (c) $S<0$; generating concave surface.

namely $S \neq 0$.

$S=\vec{B} \cdot \vec{n}=|\vec{B}||\vec{n}| \cos \theta$

(a) When $\theta=90^{\circ}, S=0$ and $\vec{B}$ and $\vec{n}$ are perpendicular to each other.

(b) When $0^{\circ} \leq \theta<90^{\circ}, S>0$ and $\vec{B}$ and $\vec{n}$ are in the same direction.

(c) When $90^{\circ}<\theta \leq 180^{\circ}, S<0$ and $\vec{B}$ and $\vec{n}$ are in the opposite direction.

The disassembly line is determined as follows. When the contour is discontinuous at singular points, such as point $\mathrm{A}$ of Fig. 6(a) and point B of Fig. 6(b), or the value $S=0$ at some points under the continuous condition such as point A of Fig. 6(c), these points are considered as the candidate points for disassembly lines. When the values $S$ of the upper portion of those points are greater than zero $(S>0)$, the first horizontal line counted from the bottom of the object is the disassembly line. For examples, point A and point B in Fig. 6(a) are two discontinuous singular points. The value $S$ in the neighborhood region above point $\mathrm{A}$ is smaller than zero. Accordingly, the horizontal line through point A (i.e. L1) is not the disassembly line. On the contrary, the horizontal line L2 through point B is the disassembly line since the value of $S$ in the neighborhood region above point B is greater than zero. For the example shown in Fig. 6(b), the value $S$ in the neighborhood region above point A is greater than zero, hence the horizontal line L1 through point A is the disassembly line while the horizontal line L2 is not the disassembly line. Similarly, the horizontal line through point A in Fig. 6(c) is the disassembly line.

\subsubsection{Disassembly rules}

Based on the definition of criterion and disassembly lines described previously, we can determine if shape converters are needed for various kinds of inserts in the fabrication process. Shape converters can be divided into two types: the simple type

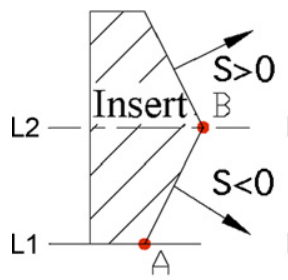

(a)

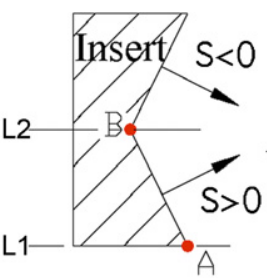

(b)

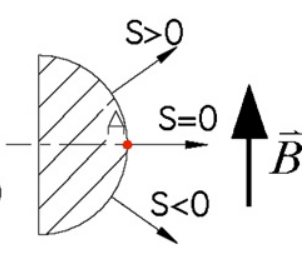

(c)
Fig. 6. Illustration of the disassembly line with respect to the value of $S$. and split type. Therefore, disassembly rules can be classified into three categories:

(1) No requirement of shape converter:

I. When the contour is parallel to insertion direction and the criterion value $S$ at any point of the contour is zero.

II. When the contour is parallel to insertion direction and the criterion value $S$ at any point of the contour is smaller than zero.

(2) Simple shape converter (no need of multiple shape converters):

I. When the contour is parallel to insertion direction and the criterion value $S$ at any point of the contour is greater than zero.

II. When the criteria values $S$ at the neighborhood region above the disassembly line are larger than or equals to zero.

(3) Split shape converter:

I. When the criterion value $S$ at any point above the disassembly line is smaller than zero.

\subsection{The fabrication procedure}

For solid insert part, it is prior to orient the insertion direction so that it is parallel to the contour of the insert when the value $S$ at any point of the contour is zero or negative. Then, the part insertion process can be completed without the assistance of shape converter. If the shape converter is necessary, simple shape converter is considered first and then the complicated split type. These guidelines are summarized in the flow chart shown in Fig. 7. The concept is explained step by step as follows:

Step 1: Judge if the RP part contains inserts or not before fabrication and the types of insert such as solid or liquid.

Step 2: If the insert is in the form of solid, the classification rules, which will be discussed in the next section, will provide the information of the requirement of shape converter, the need of multiple shape converters and the portion of the insert which will demand the shape converter. If the insert is liquid, we only require the frame of prototype to provide the room for spraying and of squeezing in the liquid materials. Next, the original laminated method is employed to complete the fabrication.

Step 3: After the frame and shape converters are manufactured, the insert or the whole set containing the insert and shape converter can be put into the frame.

Step 4: Complete the rest part of prototyping and continue the next insertion process.

\section{Verification and examples}

Three examples are demonstrated to show the feasibility of the previously described part insertion process. They are the embedded ball-joint, a calabash shape insert, and the device embedded with motor and battery as shown in Fig. 8(a-c), respectively. A new LOM prototyping approach and fabrication methods are employed for real implementation. 


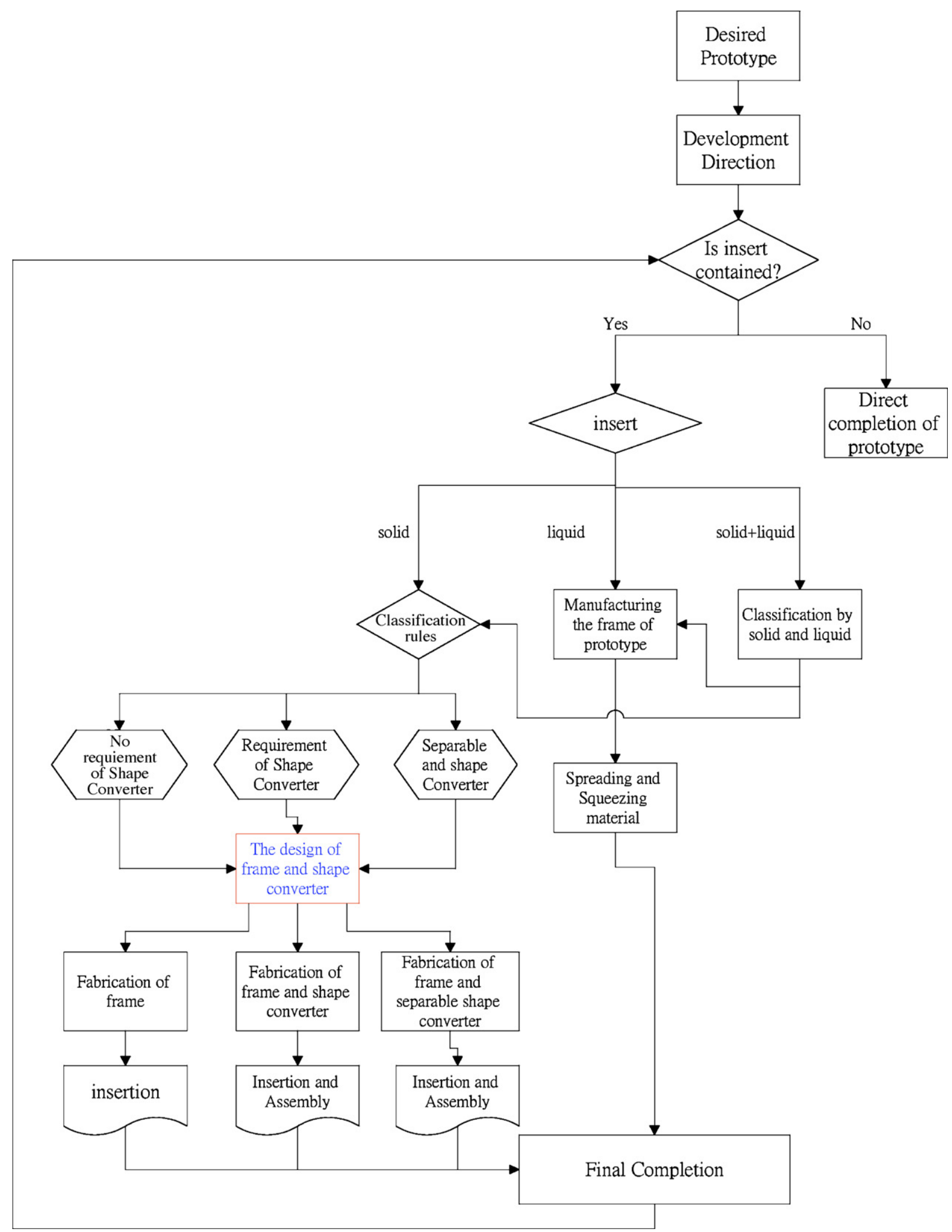

Fig. 7. The complete flow chart of insertion process.

\subsection{Case study 1-ball-joint insertion to the prototype}

It is well known that the ball-joint is composed of the ball and socket, where the socket holds the ball. This mechanical device provides certain rotational freedom. To deal with this solid balljoint insertion, its contour is analyzed and the different values of $S$ are solved. As shown in Fig. 9(a), the criterion value $S=0$ at point $\mathrm{A}$. It is less than zero for the portion beneath $\mathrm{A}$, and 


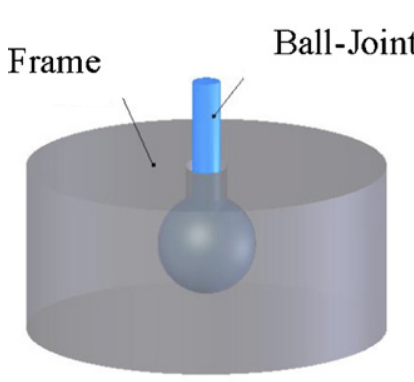

(a)

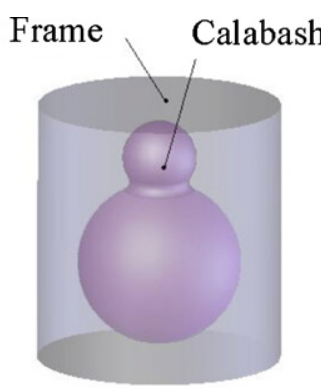

(b)

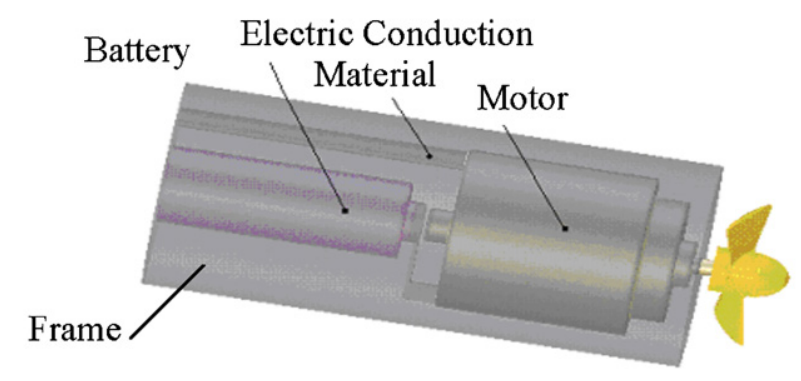

(c)

Fig. 8. (a) The ball-joint example, (b) the mock-up with calabash insert, (c) the fan mock-up with motor and battery inserts.

larger than zero for the portion above A. Hence the horizontal line through A is taken as the disassembly line, and the second classification of simple shape converter mold and frame is used as shown in Fig. 9(b).

Then, LOM is adopted to fabricate the prototype of simple shape converter and frame as shown in Figs. 10(a and b), respectively. Next, as displayed in Fig. 10(c) the ball is inserted in the frame. Finally, the shape converter is enclosed to complete the insertion process as shown in Fig. 10(d).

\subsection{Case study 2-calabash part insertion}

The calabash example will demonstrate how to combine inserts of different materials. The calculated $S$ along the contour are depicted in Fig. 11(a). Looking at this figure, it can be readily concluded that the horizontal line passes through point $\mathrm{A}$ is the disassembly line. The calabash is decomposed and the third type split shape converter and frame of the prototype as shown in Fig. 11(b) is used.

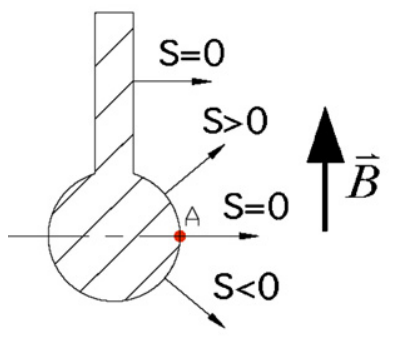

(a)

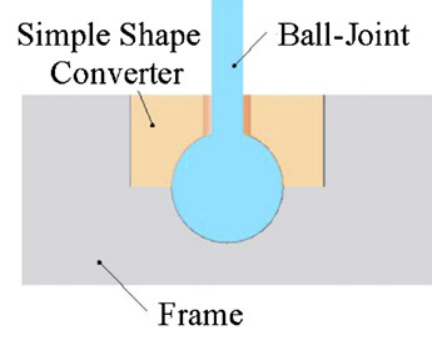

(b)

Fig. 9. (a) Determination of disassembly line, (b) the mock-up frame and simple shape converter.

(a)

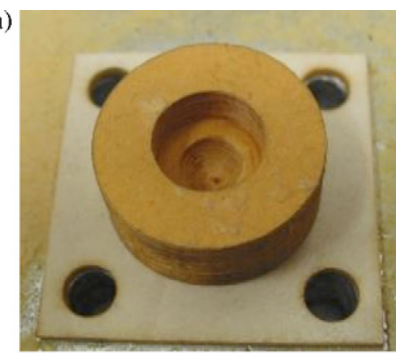

(c)

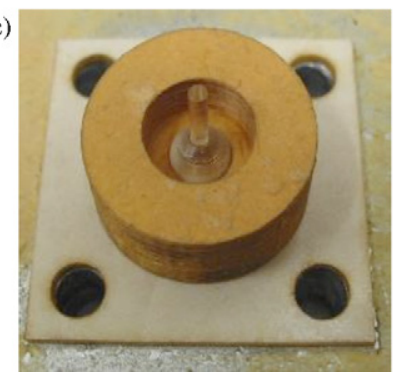

(b)

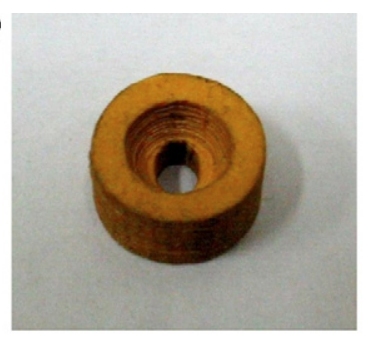

(d)

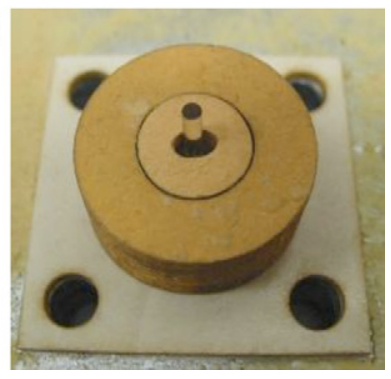

Fig. 10. Ball-joint insert process (a) frame, (b) shape converter, (c) part after ball is inserted, (d) the whole completed piece with shape converter enclosed.

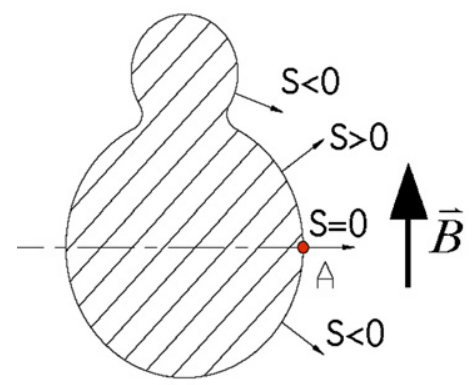

(a)
Multiple Shape Converter

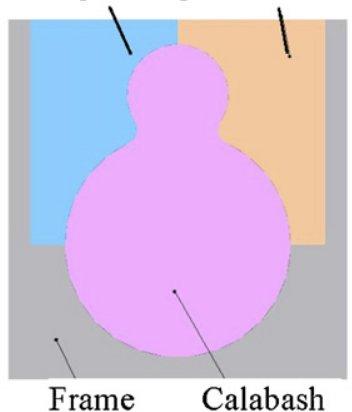

(b)

Fig. 11. (a) Determination of disassembly line, (b) the mock-up with multiple shape converter

Next, the multiple shape converter and frame are fabricated respectively, as shown in Fig. 12(a). The embedded calabash shape insert is wrapped by shape converter, and the whole set is positioned in the frame. Fig. 12(b) shows the whole prototype of built-in calabash shape item.

\subsection{Case study 3-the fan mock-up with motor and battery inserts}

In this example, the motor and battery are two embedded functional devices in the prototype. Following the disassembly

(a)
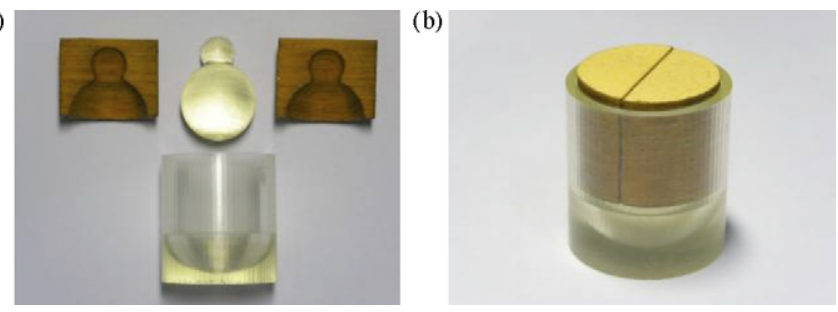

Fig. 12. The calabash shape insertion (a) shape converter and frame, (b) the completed prototype. 


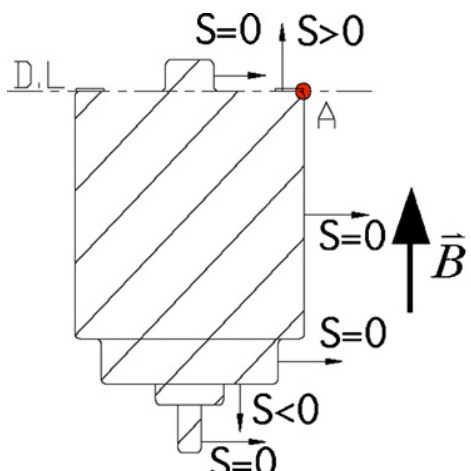

(a)

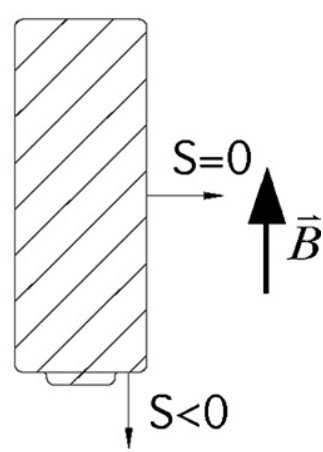

(b)
Fig. 13. Determination of disassembly lines for the motor and battery.

classification rules the contour of the motor is checked first, and the value $S$ at different part of the contour is shown in Fig. 13(a). For the region beneath the discontinuous singular point A, $S<0$ or $S=0$, while in the portion above the point A, $S>0$ or $S=0$. Clearly, the horizontal line through point A is the disassembly line. Fig. 13(b) shows the battery and its $S$ values. It is smaller or equals to zero. Hence, the first classification applies and no shape converter is required. For the motor part, the simple shape converter performs well for the most part below the disassembly line. The battery can be embedded directly as shown in Fig. 14.

However, it is noted that the second classification of simple shape converter is required for the upper portion of the motor.

After the classification, new LOM process with on-line decubing is used to fabricate the mock-up and simple shape converter separately. The initial mock-up frame for the motor

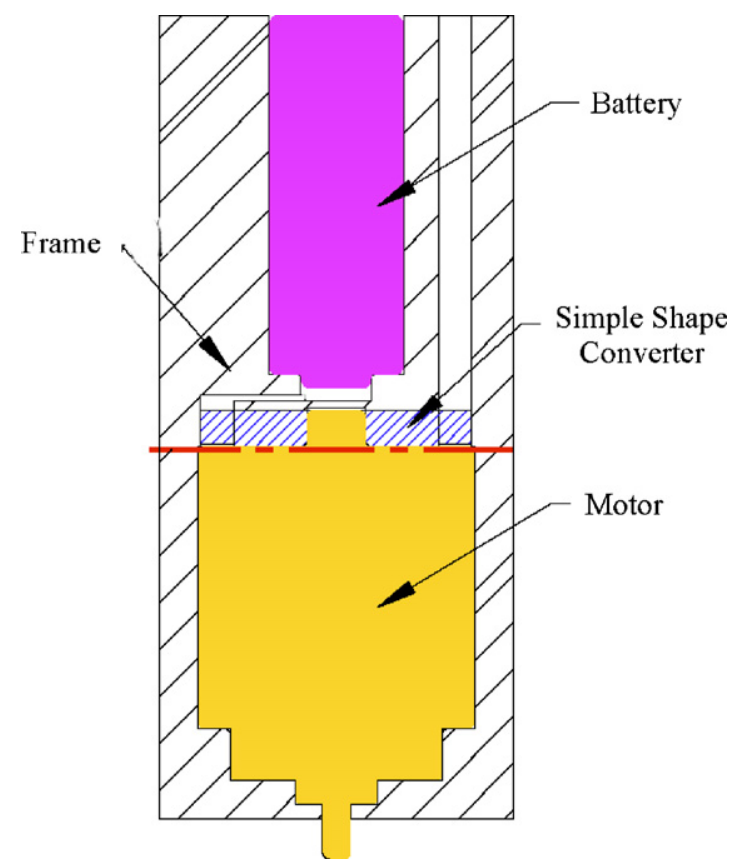

Fig. 14. The mock-up frame and the shape converter for case study 3 . (a)

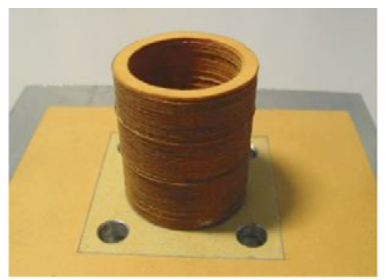

(b)

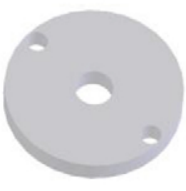

(c)

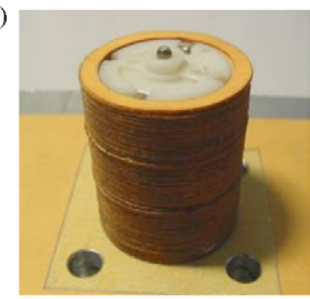

(e)

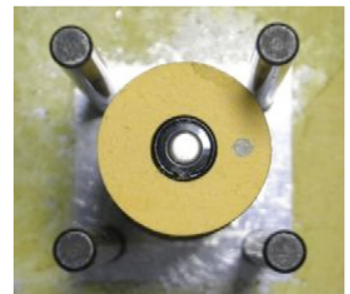

(d)

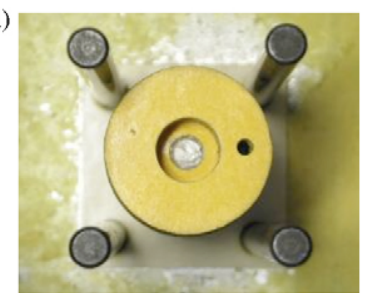

(f)

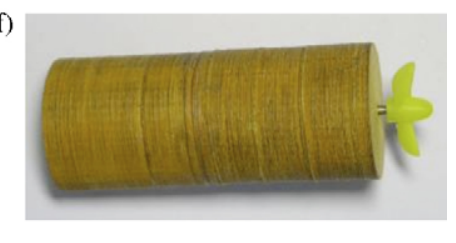

Fig. 15. The prototype of fan (a) initial frame, (b) shape converter of the motor, (c) part after motor is inserted, (d) part after shape converter is placed at the top of the motor, (e) part after battery is inserted, (f) the final prototype with built-in motor and battery.

is shown in Fig. 15(a), and the shape converter for the motor is shown in Fig. 15(b). Firstly, the motor is put into the frame (Fig. 15(c)). The shape converter is positioned above the motor as shown in Fig. 15(d), and the conductive silver glue is extruded into the hole on the left of the part. After that, the battery is inserted into the frame, and then the silver glue is extruded to the hole to complete the circuit. Finally the bottom of the battery is packed to complete this functional device. Fig. 15(e) displays the part after battery insertion and Fig. 15(f) shows the whole set of prototype with built-in motor and battery.

\section{Conclusion}

The appropriate classification rules and the fabrication procedures for making insert embedded prototype are proposed. The rules apply well for most insertion cases of symmetric functional devices such as motors, bearings and batteries. Verification via three different types of insertion examples has proved the feasibility of the proposed approach. This new development can greatly increase the application fields and values of Rapid Prototyping.

\section{References}

[1] A. Kataria, W. Rosen, Building around inserts: methods for fabricating complex devices in stereolithography, Rapid Prototyp. J. 7 (5) (2001) 253261.

[2] J. Kathryn, F.F. Kong, Procedure for rapid fabrication of non-assembly mechanisms with embedded components, in: Proceedings of the ASME 
Design Engineering Technical Conference, Montreal, Canada, September 29-October 2, 2002, pp. 1239-1245.

[3] C. Mavroidis, J. Kathryn, Fabrication of non-assembly mechanisms and robotic systems using rapid prototyping, J. Mechan. Des. 123 (4) (2001) $516-524$.

[4] L.E. Weiss, F.B. Prinz, Shape deposition manufacturing of heterogeneous structures, J. Manuf. Syst. 16 (4) (1997) 239-248.
[5] M. Hatanaka, R. Mark, Process planning for embedding flexible materials in multi-material prototypes, in: Proceedings of the ASME Design Engineering Technical Conference, vol. 3, 2003, pp. 325-333.

[6] Y.S. Liao, L.C. Chiu, A new approach of online waste removal process for laminated object manufacturing (LOM), J. Mater. Process. Technol. 140 (1-3) (2003) 136-140. 